\title{
Effect of large-scale mixing on the axisymmetric structure of turbulence correlations in complex dual-stream jets
}

\author{
M. Z. Afsar* \\ Department of Mechanical \& Aerospace Engineering, University of Strathclyde, Glasgow, GI I XJ, UK. \\ V. Gryazev ${ }^{\dagger}$ \\ School of Engineering and Material Science, Queen Mary University of London, Mile End Road, London, E1 4NS, UK.
}

\author{
A. Markesteijn ${ }^{*}$ \\ School of Engineering and Material Science, Queen Mary University of London, Mile End Road, London, E1 4NS, UK. \\ S. Karabasov $\$$ \\ School of Engineering and Material Science, Queen Mary University of London, Mile End Road, London, E1 4NS, UK.
}

\begin{abstract}
Dual-stream flows are a ubiquitous feature of turbofan engines used in civil aviation. In this paper we analyze the spatial structure of turbulence correlations in a high speed round coaxial jet operating at heated conditions. In particular we consider the effect of axisymmetry of a second rank correlation tensor and the usual fourth order Reynolds stress auto-covariance tensor that enters the Goldstein's generalized acoustic analogy formulation. The invariants of these tensors can be reduced to a simpler form depending on whether isotropy or axisymmetry was assumed. We show that an axisymmetric turbulence approximation remains accurate in the core region but tends to break down in the bypass stream and especially in the interfacial region between both streams where high level of mixing of turbulence takes place. In the paper we present some of our latest results and provide a road map for the future calculations that we have planned.
\end{abstract}

\section{Introduction}

$\mathrm{T}$ HE flow from an exhaust nozzle in a turbofan engine used in modern civil aviation possess two co-axial streams. The interaction between the main core and bypass streams leads to a highly complex turbulence structure, which is likely to be more pronounced when the operating point (OP) of the engine is supersonic and heated. The promotion of large-scale mixing of the initial shear layers along the nozzle lip lines from the dual stream interacting with core flow naturally has an impact on the far-field radiation. One of the most effective ways to assess this is via Aero-acoustic prediction model based on Goldstein's generalized acoustic analogy formulation. The generalized analogy formulation shows that in the absence of solid surface effects and unheated conditions, the acoustic spectrum formula of a jet flow depends on the near field turbulence through the Reynolds stress auto-covariance tensor, $R_{i j k l}(\boldsymbol{y}, \boldsymbol{\eta}, \tau)$. Here, the tensor suffixes range from $(i, j, k, l)=(1,2,3)$ corresponding to the velocity perturbation relative to the Favre (density-weighted) averaged mean flow along Cartesian directions. This tensor is a vector function of position, $\boldsymbol{y}$, as well as spatial separation, $\boldsymbol{\eta}$, and time delay, $\tau$, between two correlated points in the jet.

As we shall see in $\S .3$, For the general heated flow the 2nd and 4th suffix of the above tensor range from $(1,2,3,4)$ and are denoted by Greek letters. Modeling the space-time structure of this tensor still remains a daunting task because, in general, it possesses 144 components, but not all of these are independent. Afsar et al. (2011) showed that symmetry between tensor suffixes, reduces (without approximation) to 63 independent components, which is still too large for practical use especially in complex flow conditions of a dual-stream nozzle. However since the nozzle is ostensibly round the most natural approximation for the turbulence structure of the Reynolds stress auto-covariance (or the generalized stress tensor) would be to assume that it is an axisymmetric tensor. While this has been investigated before using LES data (see Afsar et al. 2010 \& 2011 and Kreitzman \& Nichols 2015 who applied the Afsar et al. 2010 analysis to a

\footnotetext{
*Chancellor's Fellow. AIAA Member.

$\dagger$ PhD Candidate. AIAA Member.

${ }^{\ddagger}$ Research Scientist / Director, GPU-prime Ltd. AIAA Member.

${ }^{\S}$ Reader in Computational Modelling. AIAA Senior Member.
} 


$\begin{array}{lllll}\text { Operating Point (OP) } & \text { Description } & M_{J} & T R & M a \\ \text { OP 1.3 } & \text { Dual stream heated ideally expanded } & 0.86 & 2.7 & 1.4 \\ \text { OP 1.7 } & \text { Single stream unheated subsonic } & 0.64 & 1.0 & 0.64\end{array}$

Table 1 Gryazev et al (2019) [1] test cases

chevron jet), there has not been any systematic investigation of the structure of high-order turbulence correlations pertaining to the Reynolds stress auto-covariance, or its generalized form, in the complex jet scenario shown in Fig. 1 below. The aim of this paper is, therefore, to conduct such a study with the aim of: (1). assessing the validity of the axisymmetric turbulence approximation developed in Afsar et al.(2011) and (2). examining several non-axisymmetric approximations for the generalized stress tensor in Goldstein's acoustic analogy.

We use data obtained from a Large-Eddy Simulations of a high Reynolds number $\left(O\left(10^{6}\right)\right)$ heated and non-heated co-axial round jet flow that operates at conditions shown in table 1 . The large-eddy simulation data was reported in previously in Gryazev et al. (2019) and involves two static single-stream co-axial jets as depicted in Fig. 1. The LES calculation is based on the Monotonically Integrated LES (MILES) approach. The calculation domain includes the axi-symmetric nozzle geometry as well as the jet flow with grid volume of almost 20 million cells of the implicit-multi-block hexagonal type. For the calculations, the high-resolution CABARET method was used (see Semiletov et al. [2]).

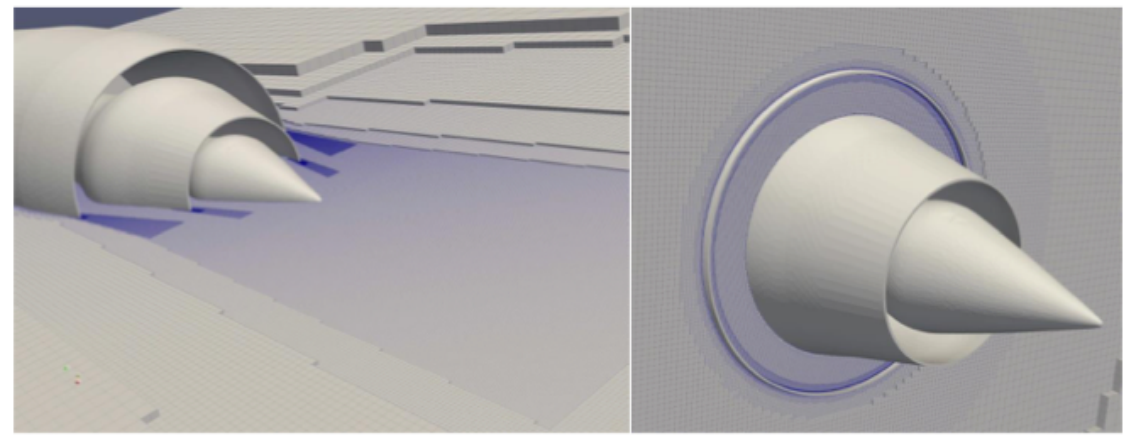

Fig. 1 Dual stream structure of flow. Three-dimensional view of the LES grid (reproduced from Fig. 1 in [1])

\section{Definitions, symmetries and approximations}

Consider a region of non-homogeneous turbulence bounded within a high speed jet of order-1 acoustic Mach number, $M a=U_{J} / c_{\infty}$ and order-1 temperature ratio, $T R$. Pressure fluctuations within the jet propagate to the far field where they are perceived as sound. We use Goldstein's generalized acoustic analogy [3] to represent this process in a manner whereby the wave propagation is calculated via a propagator tensor that depends on ALEE solution and the Reynolds stress auto-covariance tensor. The latter is modeled appropriately (see appendix). Let the pressure $p$, density $\rho$, enthalpy $h$, and speed of sound $c$ satisfy the ideal gas law equation of state $p=\rho c^{2} / \gamma$ and $h=c^{2} /(\gamma-1)$, where $\gamma$ denotes the ratio of specific heats. 
The acoustic spectrum at the observation point, $\boldsymbol{x}=\left(x_{1}, \boldsymbol{x}_{T}\right)=\left(x_{1}, x_{2}, x_{3}\right)$, given by the Fourier transform

$$
I(\boldsymbol{x}, \omega) \equiv \frac{1}{2 \pi} \int_{-\infty}^{\infty} e^{i \omega \tau} \overline{p^{\prime}(\boldsymbol{x}, t) p^{\prime}(\boldsymbol{x}, t+\tau)} d \tau,
$$

of the far-field pressure auto-covariance, $\overline{p^{\prime}(\boldsymbol{x}, t) p^{\prime}(\boldsymbol{x}, t+\tau)}$, can be expressed as a volume integral over a unit volume of turbulence at $\boldsymbol{y}=\left(y_{1}, \boldsymbol{y}_{T}\right)=\left(y_{1}, y_{2}, y_{3}\right)$ in the jet via

$$
I(\boldsymbol{x} ; \omega)=\int_{V_{\infty}(\boldsymbol{y})} I(\boldsymbol{x}, \boldsymbol{y} ; \omega) d \boldsymbol{y},
$$

where, $V_{\infty}(\boldsymbol{y})$ is the entire source region.

The pressure fluctuation in 11 is defined as $p^{\prime}(\boldsymbol{y}, \tau) \equiv p(\boldsymbol{y}, \tau)-\bar{p}(\boldsymbol{y})$ where over-bars are denote time average,

$$
\bar{\bullet}(\boldsymbol{x}) \equiv \lim _{T \rightarrow \infty} \frac{1}{2 T} \int_{-T}^{T} \bullet(x, t) d t
$$

such that $\bullet$ in 3 is a place holder for any fluid mechanical variable, $T$ is the time period of averaging and, by definition, $\overline{\bullet^{\prime}}=0$.

Goldstein \& Leib (2008; hereafter referred to as G \& L) showed that $I(\boldsymbol{x}, \boldsymbol{y} ; \omega)$ on right side of (2) depends on the turbulence through the scripted tensor, $\mathcal{H}_{\lambda j \mu l}(\boldsymbol{y}, \boldsymbol{\eta} ; \omega)$, which is related to the Fourier transform

$$
H_{\lambda j \mu l}(\boldsymbol{y}, \boldsymbol{\eta} ; \omega)=\frac{1}{2 \pi} \int_{-\infty}^{\infty} e^{i \omega \tau} R_{\lambda j \mu l}(\boldsymbol{y}, \boldsymbol{\eta} ; \tau) d(\tau)
$$

of the generalized auto-covariance tensor,

$$
R_{\lambda j \mu l}(\boldsymbol{y}, \boldsymbol{\eta} ; \tau) \equiv \lim _{T \rightarrow \infty} \frac{1}{2 T} \int_{-T}^{T} e_{\lambda j}(\boldsymbol{y}, \tau) e_{\mu l}\left(\boldsymbol{y}+\boldsymbol{\eta}, \tau+\tau_{0}\right) d \tau_{0}
$$

of the stationary random function, $e_{\lambda j}(\boldsymbol{y}, \tau)=-\left[\rho v_{\lambda}^{\prime} v_{j}^{\prime}-\overline{\rho v_{\lambda}^{\prime} v_{j}^{\prime}}\right](\boldsymbol{y}, \tau)$, by the linear transformation $\mathcal{H}_{\lambda j \mu l}(\boldsymbol{y}, \boldsymbol{\eta} ; \omega):=$ $\epsilon_{\lambda j \sigma m} H_{\sigma m \gamma n}(\boldsymbol{y}, \boldsymbol{\eta} ; \omega) \epsilon_{\mu l \gamma n}$. Comparing (5.12) to (5.13) in G \& L (2008) and using appropriate outer products of unit tensors in suffixes $(\lambda, j, \sigma, m)$ allows definition of the tensor as, $\epsilon_{\lambda j \sigma m} \equiv \delta_{\lambda \sigma} \delta_{j m}-\delta_{\lambda j} \delta_{\sigma m}(\gamma-1) / 2$ in the linear relation for $\mathcal{H}_{\lambda j \mu l}$ above. The four-dimensional perturbation velocity, $v_{\lambda}^{\prime}(\boldsymbol{y}, \tau) \equiv v_{\lambda}(\boldsymbol{y}, \tau)-\tilde{v}_{\lambda}(\boldsymbol{y})$ in which $v_{\lambda}^{\prime}=v_{i}^{\prime}$ is the ordinary fluid velocity perturbation when suffix, $\lambda=i=(1,2,3)$, otherwise $v_{\lambda}^{\prime}=v_{4}^{\prime}$ is proportional to enthalpy fluctuation. The latter denotes $v_{4}^{\prime}:=(\gamma-1)\left(h^{\prime}+v^{\prime 2} / 2\right) \equiv\left(c^{2}\right)^{\prime}+(\gamma-1) v^{\prime 2} / 2$ where $h^{\prime}$ is the fluctuating static enthalpy and $\left(c^{2}\right)^{\prime}$ is the fluctuations in the sound speed squared such that $v_{4}^{\prime} /(\gamma-1)$ denotes the moving frame stagnation enthalpy fluctuation (discussed further in [4]).

The tensor $R_{\lambda j \mu l}\left(y, \eta_{1}, \eta_{\perp} ; \tau\right)$ possesses 144 components $(3 \times 4 \times 3 \times 4)$, however, owing to its two pair symmetry property - inasmuch as $R_{i j k l}=R_{j i k l}$ and $R_{i j k l}=R_{i j l k}$ when $(\lambda, \mu)=(1,2,3)-$ not all of these are independent. Afsar et al. (2011) (see table 1 on p.2525 of their paper) show that 144 reduces to 63 independent components when these symmetries are taken into account. Further reducing this number of components then involves achieving an "irreducible representation" of the tensor using its symmetry approximations, such as isotropy for example.

In this paper, we use an axisymmetric turbulence model that is a much more realistic kinematic representation for jets and which reduces the 63 components to a manageable number. The approximation assumes that the transverse correlation lengths are small compared to that in the streamwise flow direction. This is a well founded assertion in jets (see, for example, Pokora \& McGuirk's measurements[] in Figs. 19-21 and also Fig.10 of their conference paper, AIAA 2008-3028). Afsar et al. (2011) used Pokora \& McGuirk's data to propose that $R_{\lambda j \mu l}\left(\boldsymbol{y}, \eta_{1}, \eta_{\perp} ; \tau\right)$ is an axisymmetric tensor where $\eta_{\perp}=\left|\boldsymbol{\eta}_{\perp}\right|$ and $\boldsymbol{\eta}_{\perp}=\left(\eta_{2}, \eta_{3}\right)$. The spectral equivalent of this (lemma's 3.1 and 3.2 in Afsar[5]) requires that $\Phi_{\lambda j \mu l}^{*}\left(y, k_{1}, k_{\perp}^{2} ; \omega\right)$ is axisymmetric with the streamwise direction, $k_{1}$, being the principle direction of invariance. The physical space approximation is consistent with experiments by Morris \& Zaman [6] who show in their Fig. 15 that the 
transverse and azimuthal correlation lengths are virtually constant across range, $S t=(0.01-1.0)$ for an isothermal axisymmetric jet. The real space (i.e. non-spectral tensor) equivalent of Eq. (3.20) in reference [5] is:

$$
\begin{aligned}
R_{i j k l}\left(\boldsymbol{y}, \eta_{1}, \eta_{\perp}, \tau\right) & =\left[\delta_{i j} \delta_{k l}-\delta_{i 1} \delta_{j 1} \delta_{k l}-\delta_{k 1} \delta_{l 1} \delta_{i j}+\delta_{i 1} \delta_{j 1} \delta_{k 1} \delta_{l 1}\right] R_{2222}\left(\boldsymbol{y}, \eta_{1}, \eta_{\perp}, \tau\right) \\
& +\left[\delta_{i k} \delta_{j l}+\delta_{i l} \delta_{j k}-2 \delta_{i j} \delta_{k l}+2 \delta_{i 1} \delta_{j 1} \delta_{k l}+2 \delta_{k 1} \delta_{l 1} \delta_{i j}\right. \\
& \left.-\delta_{i 1} \delta_{l 1} \delta_{j k}-\delta_{j 1} \delta_{l 1} \delta_{i k}-\delta_{j 1} \delta_{k 1} \delta_{i l}-\delta_{i 1} \delta_{k 1} \delta_{j l}\right] R_{2323}\left(\boldsymbol{y}, \eta_{1}, \eta_{\perp}, \tau\right) \\
& +\left[\delta_{i 1} \delta_{l 1} \delta_{j k}+\delta_{j 1} \delta_{l 1} \delta_{i k}+\delta_{j 1} \delta_{k 1} \delta_{i l}\right. \\
& \left.+\delta_{i 1} \delta_{k 1} \delta_{j l}-4 \delta_{i 1} \delta_{j 1} \delta_{k 1} \delta_{l 1}\right] R_{1212}\left(\boldsymbol{y}, \eta_{1}, \eta_{\perp}, \tau\right) \\
& +\left[\delta_{i 1} \delta_{j 1} \delta_{k l}-\delta_{i 1} \delta_{j 1} \delta_{k 1} \delta_{l 1}\right] R_{1122}\left(\boldsymbol{y}, \eta_{1}, \eta_{\perp}, \tau\right) \\
& +\left[\delta_{k 1} \delta_{l 1} \delta_{i j}-\delta_{i 1} \delta_{j 1} \delta_{k 1} \delta_{l 1}\right] R_{2211}\left(\boldsymbol{y}, \eta_{1}, \eta_{\perp}, \tau\right) \\
& +\delta_{i 1} \delta_{j 1} \delta_{k 1} \delta_{l 1} R_{1111}\left(\boldsymbol{y}, \eta_{1}, \eta_{\perp}, \tau\right)
\end{aligned}
$$

\begin{tabular}{|c|c|c|}
\hline & $\begin{array}{l}\text { DLAGONAL QUADRATIC FORM } \\
\qquad \frac{R_{i i, k k}(\underline{y}, \underline{0}, 0)}{R_{11,11}(\underline{y}, \underline{0}, 0)}\end{array}$ & $\begin{array}{l}\text { HERMITIAN QUADRATIC FORM } \\
\qquad \frac{R_{i k, i k}(\underline{y}, \underline{0}, 0)}{R_{11,11}(\underline{y}, \underline{0}, 0)}\end{array}$ \\
\hline $\begin{array}{l}\text { Isotropy } 1 \\
\text { (Afsar et al. 2007) }\end{array}$ & 5 & 5 \\
\hline $\begin{array}{l}\text { Isotropy } 2 \\
\text { (G \& L) }\end{array}$ & $9-12 \frac{R_{12,12}}{R_{11,11}}$ & $3+6 \frac{R_{12,12}}{R_{11,11}}$ \\
\hline $\begin{array}{l}\text { Axisymmetry } \\
\text { (Eq. 3.20, Afsar } \\
\text { 2012) }\end{array}$ & $1+\frac{4}{R_{11,11}}\left[R_{22,22}-R_{23,23}+R_{11,22}\right]$ & $1+\frac{2}{R_{11,11}}\left[R_{22,22}+2 R_{12,12}+R_{23,23}\right]$ \\
\hline
\end{tabular}

As discussed in Afsar et al. (2010), the tensor $R_{i j k l}$ possesses two invariants quadratic forms: $R_{i j i j}$ and $R_{i i j j}$. The validity of (6) can be assessed by contracting $R_{i j k l}$ to $R_{i j i j}$ and $R_{i i j j}$ using the LES data to compute the terms on the right hand sides without approximation and comparing that to reduced formulae obtained for these invariants using (6). The reduced formulae are summarized in Fig. 2 below. Following [7] we compare to other simpler kinematic models of $R_{i j k l}$ such as the statistical isotropic model given by Eq. (34) in Afsar et al. [8] and Eqs. (6.11) \& (6.12) in [9].

Fig. 2 Reduced form of the invariants of $R_{i j k l}$ (Table 1 in [7]) 


\section{Amplitude results of the invariants of $R_{i j k l}$}

Figs. 3 and 4 show the spatial structure of $R_{i i j j}$ and $R_{i j i j}$ at the radial shear layer location of core stream $\left(r / D_{j}=0.18\right)$ and for the bypass stream $\left(r / D_{j}=0.38\right)$ for OP1.3. It is clear that axi-symmetry given by (6) does perform very well at the these locations. There is some departure at large streamwise distances $x / D_{j}>6$ for $R_{i j i j}$ at the location of the core stream, which could be an artefact of the large-scale mixing effect alluded to in the Introduction. While there does not appear to be any appreciable departure for the streamwise structure of the invariants in the bypass stream, there is some evidence of this in Fig. 4, which shows the structure of the Millionshchikov identity. That is, (6) implies that, $R_{2222}=R_{2233}+2 R_{2323}$ (equation 12.131 on p.68 of Monin \& Yaglom [10]).

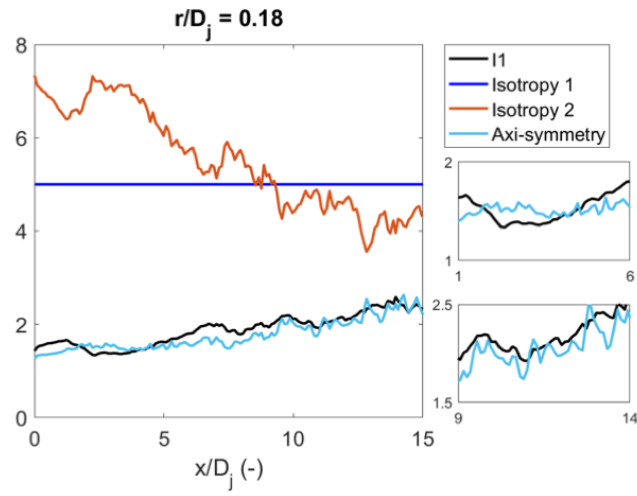

(a) Invariant $1, R_{i i j j}$ in core stream

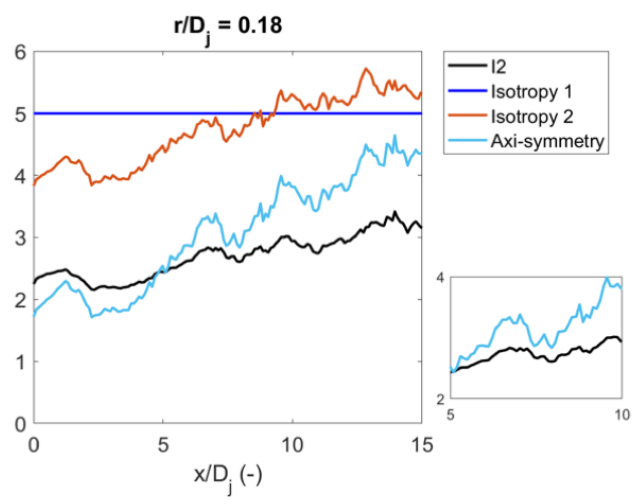

(c) Invariant $2, R_{i j i j}$ in core stream

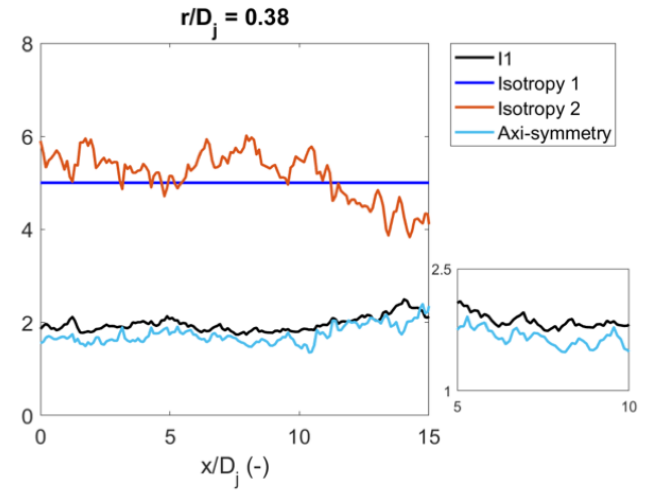

(b) Invariant $1, R_{i i j j}$ in bypass stream

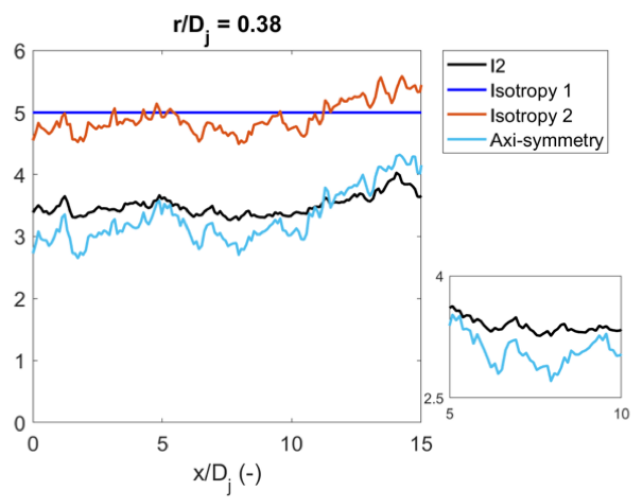

(d) Invariant $2, R_{i j i j}$ in bypass stream

Fig. 3 Streamwise structure of invariants in Fig. 2.

\section{A. Radial variation of amplitudes of Invariants}

In Figs. 5-8 we show the radial variation of the invariants in Fig.2 (Figs. 5-7) and the Millionshchikov identity (Fig. 8). In the main axi-symmetry (defined through either Invariants 1, 2 or the Millionschikov identity) is accurate within the jet region. That is for $r / D_{j}<1$. This is the case for the streamwise location that is closest to the nozzle (Figs. 5a-b and 6a-b), i.e. $x / D_{j}=2$. Interestingly, at locations further away, near the long region that defines the end of the potential core $\left(6<x / D_{j}<10\right)$ the agreement is even better and shows that axi-symmetric turbulence defined by the relations in Fig. 2 hold true up to $r / D_{j} \sim 1.5$. Similar results are found with the Millionshchikov identity in Fig. 8 (cf. Figs. 8a-b to Figs. 8c-d). 


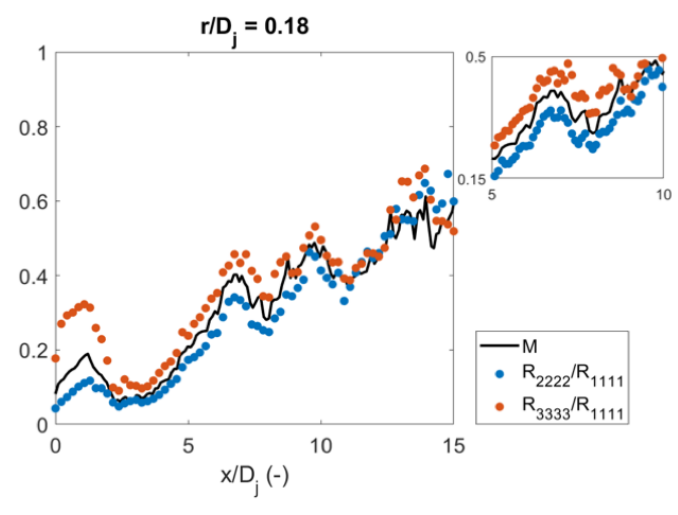

(a) M identity in core stream

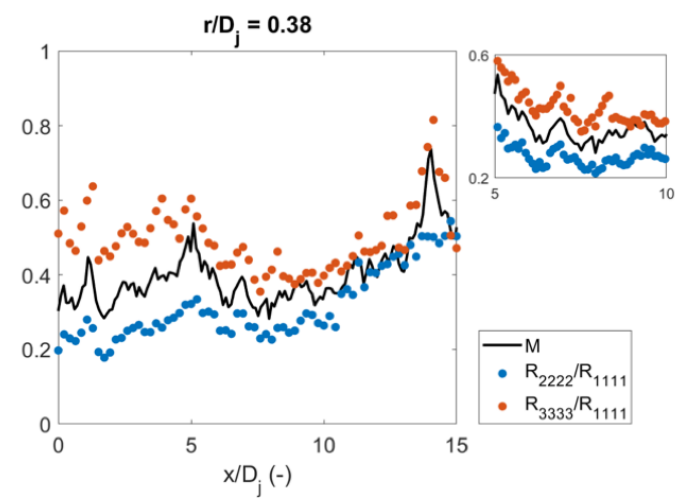

(b) $\mathrm{M}$ identity in bypass stream

Fig. 4 Streamwise structure of the Millionshchikov identity.

\section{Space-time structure of axi-symmetric turbulence}

One aspect of the validation of the basic axi-symmetric turbulence theory that was never considered before was the variation of the relations in Fig. 2 with spatial separation and/or time delay (the analysis in [5] only considered the amplitude). In Figs. 9-11 we conduct the first step toward achieving this goal by showing how the Millionshchokov identity varies with non-dimensional time delay at various fixed values of streamwise separation (we considered $d x / D j=(0.1,0.3)$ at the fixed streamwise locations where the turbulence is at its most intense (i.e. at $\left.x / D_{j}=(6,10)\right)$. Comparing Figs. 10 to 9, we see that the turbulence within the core region of OP1.3 remains relatively axi-symmetric (i.e. $R_{2222}=R_{3333}$ here and the Millionschikov identity holds) for at least half of the range of time delays that we considered. In the bypass region of OP1.3 however (Fig. 10), as the spatial separation is increased (Figs. 10b \& d) the depature from axi-symmetry appears to increase further compared to core region in Figs.9b \& $9 \mathrm{~d}$ respectively. The bypass flow for OP1.3 captures the features of axi-symmetric turbulence stated above for almost all values of time delay that we considered and with increasing streamwise separation.

\section{Conclusion}

The aim of this paper was to assess what impact a complex axisymmetric flow field such as that produced by supersonic heated coaxial flow has on the turbulence structure of high order turbulence correlations that determine the sound radiation in the acoustic analogy. This validation was not conducted before for the co-axial heated flow operating at high jet speeds Our results indicate that the large-scale mixing induced by the nozzle operating in the complex flow condition for OP1.3 within which a core region interacts across an interface with the bypass flow changes the structure of what initial (in the core region at least — see Fig. 9) is more-or-less axi-symmetric. The fact that the OP1.7 (see Fig. 11) operating point does display axi-symmetric turbulence qualities gives credence to the above hypothesis. Future work will consider the space-time structure of the invariants in Fig. 2 as well as their structure in the frequency domain.

\section{Acknowledgments}

Computational resources from HPC2, Mississippi State University, are appreciated. MZA would like to thank Strathclyde University for financial support from the Chancellor's Fellowship..

\section{References}

[1] Gryazev, V., Markesteijn, A. P., and Karabasov, S. A., "Low-Order models of dual-stream jet noise with temperature effects based on the Goldstein generalised acoustic analogy," 25th AIAA Aeroacoustics Conference, 2019.

[2] Semiletov, V. A., Karabasov, S. A., and Markesteijn, A. P., "Empiricism-free noise calculation from LES solution based on Goldstein generalized acoustic analogy: volume noise sources and mean flow effects," 21st AIAA/CEAS Aeroacoustics Conference, 2015. 
[3] Goldstein, M. E., “A generalized acoustic analogy,” J. Fluid Mech., Vol. 488, 2003, p. 315.

[4] Afsar, M. Z., Sescu, A., and Sassanis, V. G., "Effect of non-parallel mean flow on the acoustic spectrum of heated supersonic jets: Explanation of "jet quietening"," Phys. Fluids, Vol. 31, 105107, 2019.

[5] Afsar, M. Z., "Asymptotic properties of the overall sound pressure level of sub-sonic air jets using isotropy as a paradigm," J. Fluid Mech., Vol. 664, 2010, p. 510.

[6] Morris, P. J., and Zaman, K., "Velocity measurements in jets with application to noise source modeling," J. Sound \& Vib., Vol. 329, 2010, p. 394.

[7] Afsar, M. Z., Pokora, C. D., and McGuirk, J. J., "Statistical axisymmetric model of the two-point time delayed auto-covariance of the Reynolds stress tenso," 19th Polish Fluid Mechanics Conference, Poznan, Poland, 2010.

[8] Afsar, M. Z., Dowling, A. P., and Karabasov, S. A., "Jet noise in the 'zone of silence'," 13th AIAA Aeroacoustics Conference, 2007.

[9] Goldstein, M. E., and Leib, S. J., “The Aero-acoustics of slowly diverging supersonic jets,” J. Fluid Mech., Vol. 488, 2008 , p. 315.

[10] Monin, A. S., and Yaglom, A. M., Statistical fluid mechanics, vol II, chapter and pages. 

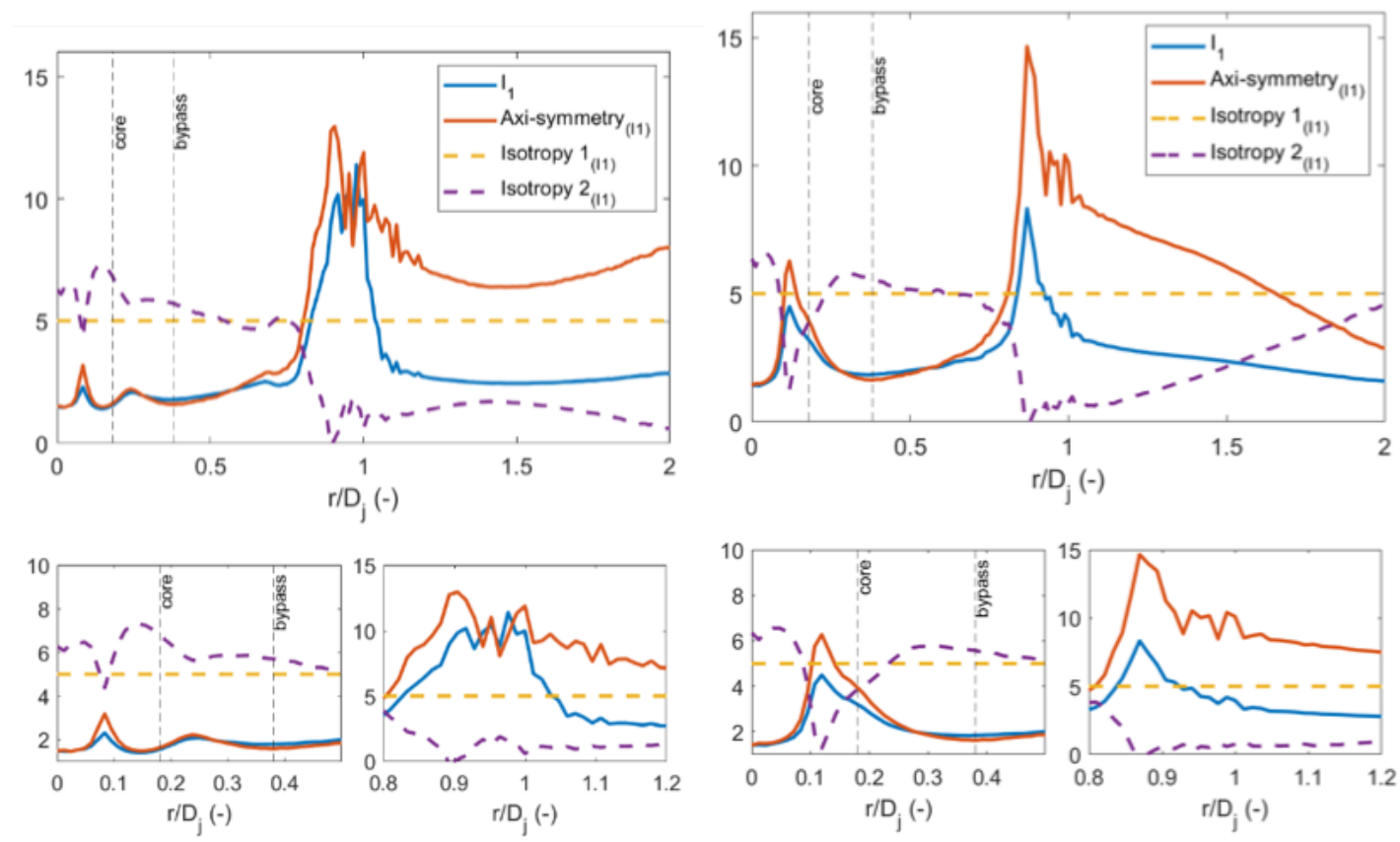

(a) $x / D_{j}=2$ : OP1.3

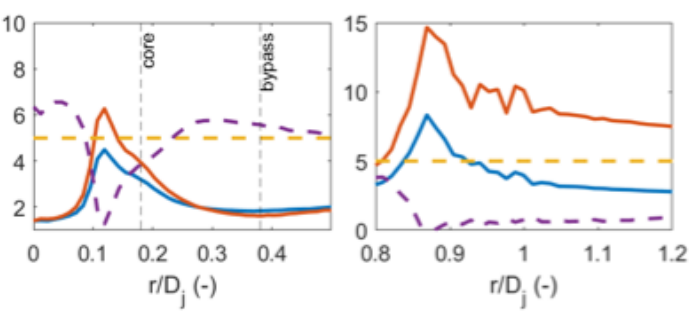

(b) $x / D_{j}=2$ : OP1.7
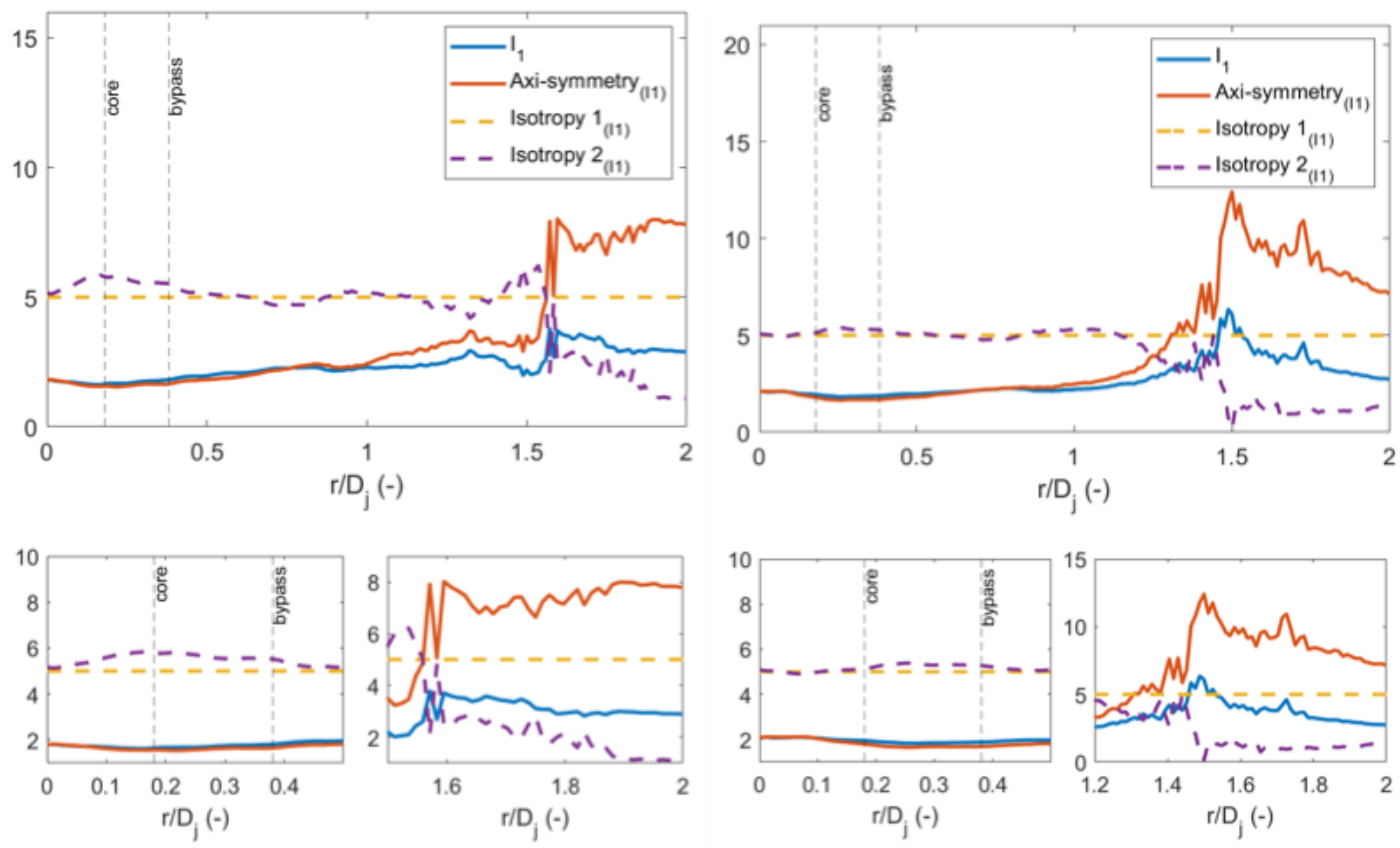

(c) $x / D_{j}=6, \mathrm{OP} 1.3$

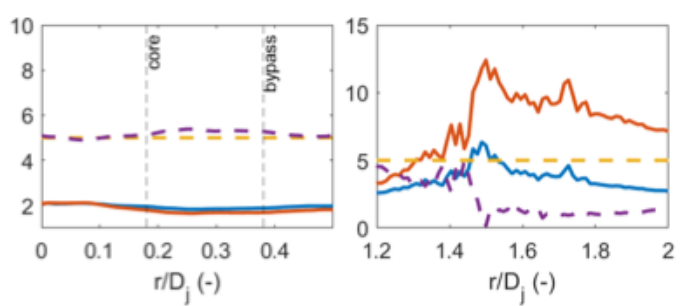

(d) $x / D_{j}=6$, OP1.7

Fig. 5 Radial variation of invariant 1 in Fig. 2 showing core and bypass regions. 

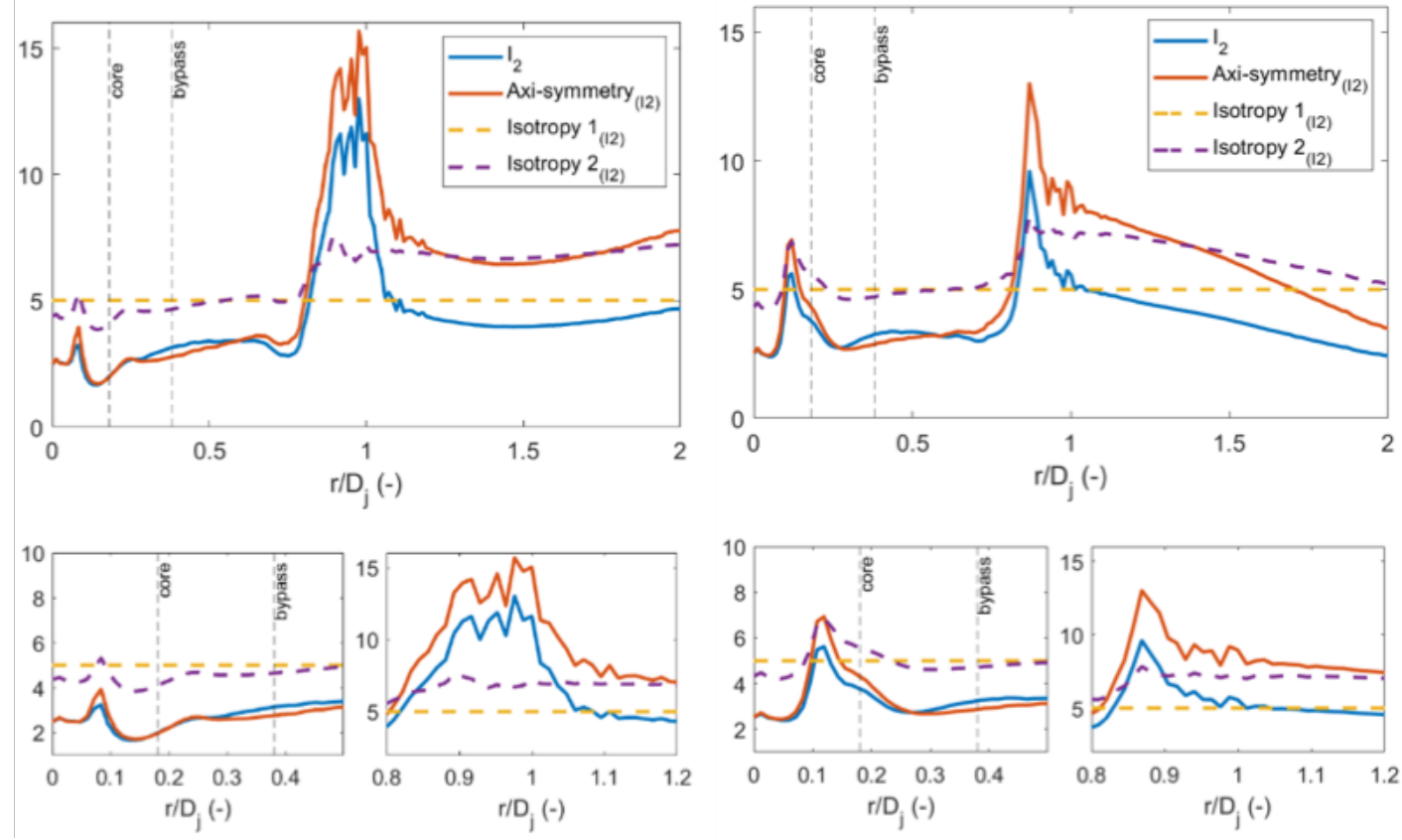

(a) $x / D_{j}=2$ : OP1.3

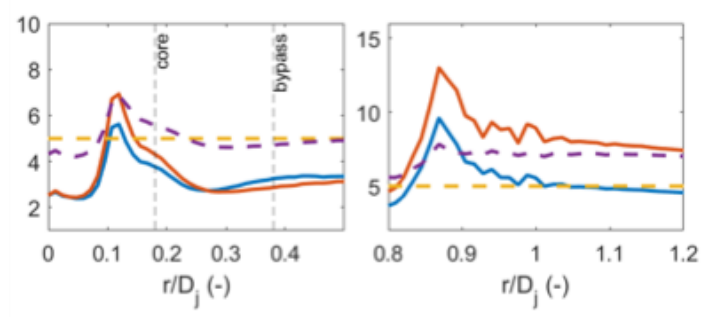

(b) $x / D_{j}=2: \mathrm{OP} 1.7$
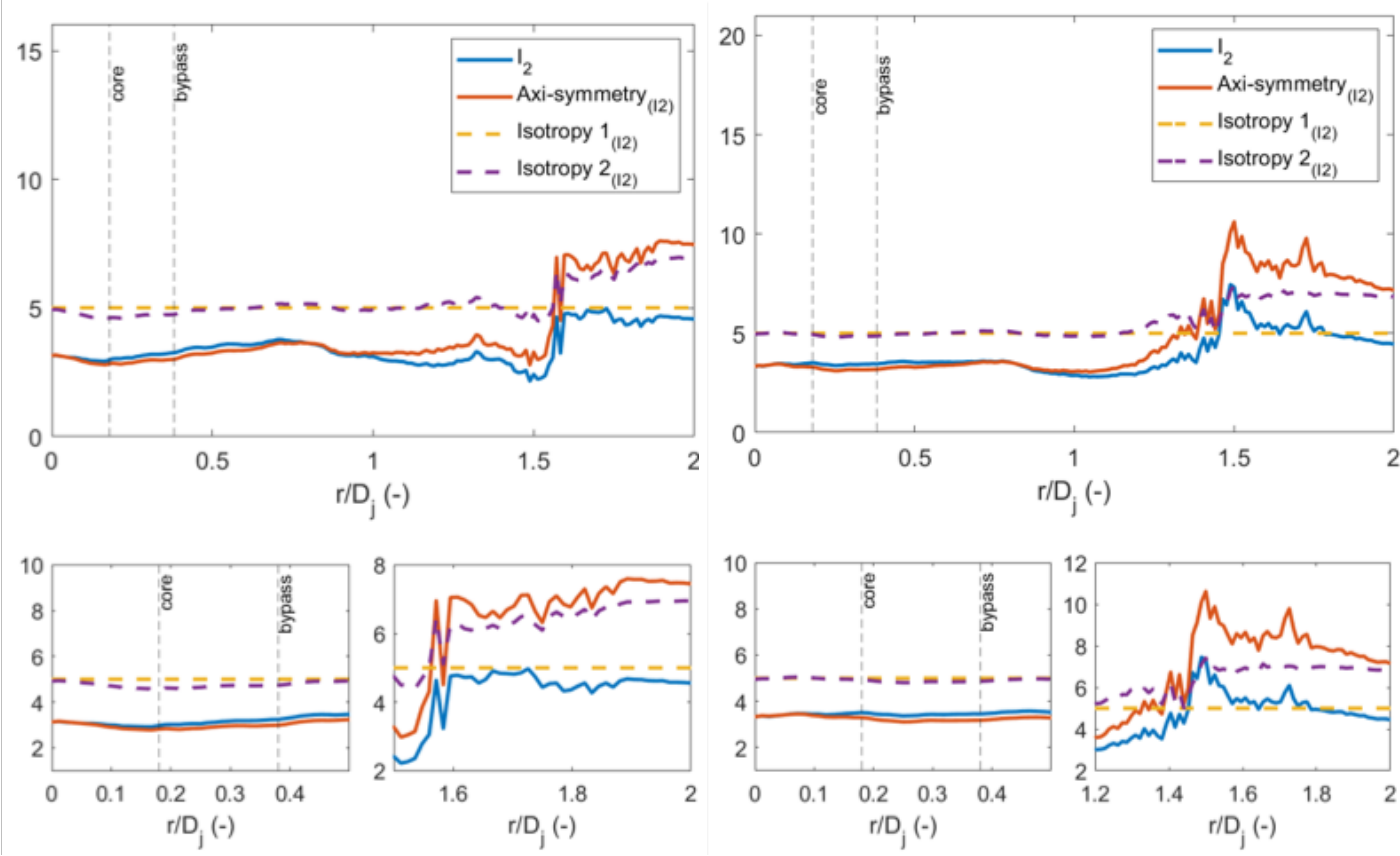

(c) $x / D_{j}=6, \mathrm{OP} 1.3$

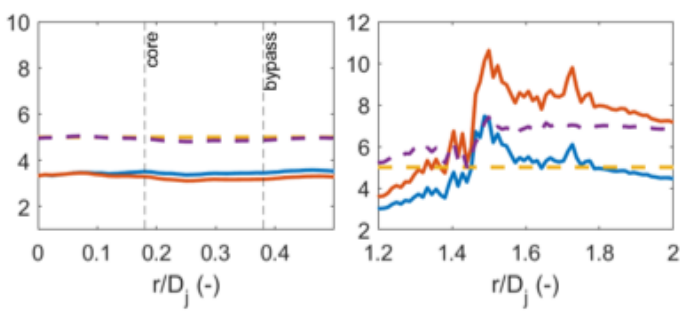

(d) $x / D_{j}=6, \mathrm{OP} 1.7$

Fig. 6 Radial variation of invariant 2 in Fig. 2 showing core and bypass regions. 

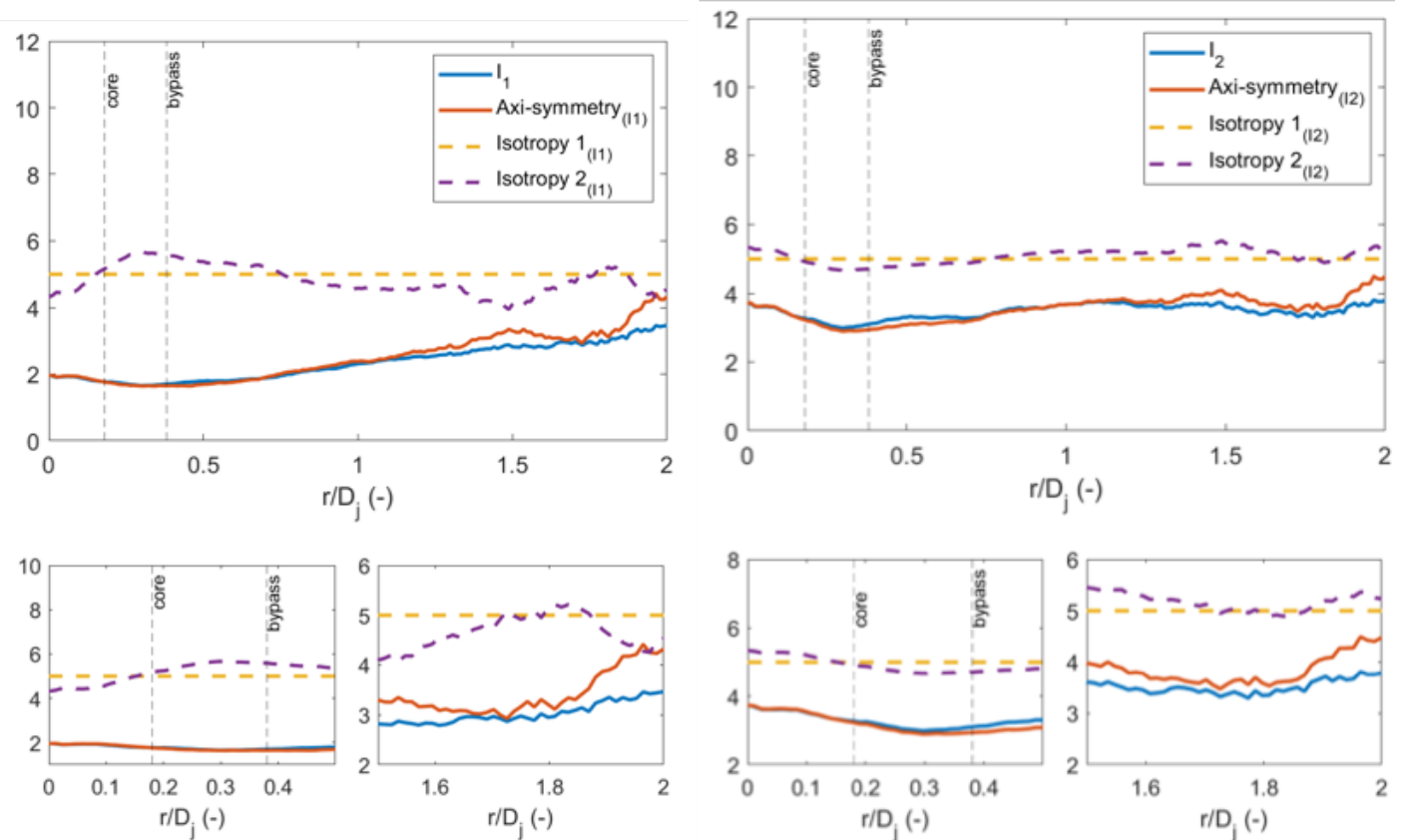

(a) Invariant 1: OP1.3

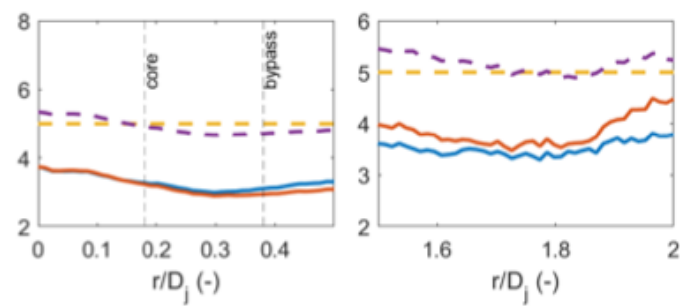

(b) Invariant 2: OP1.3
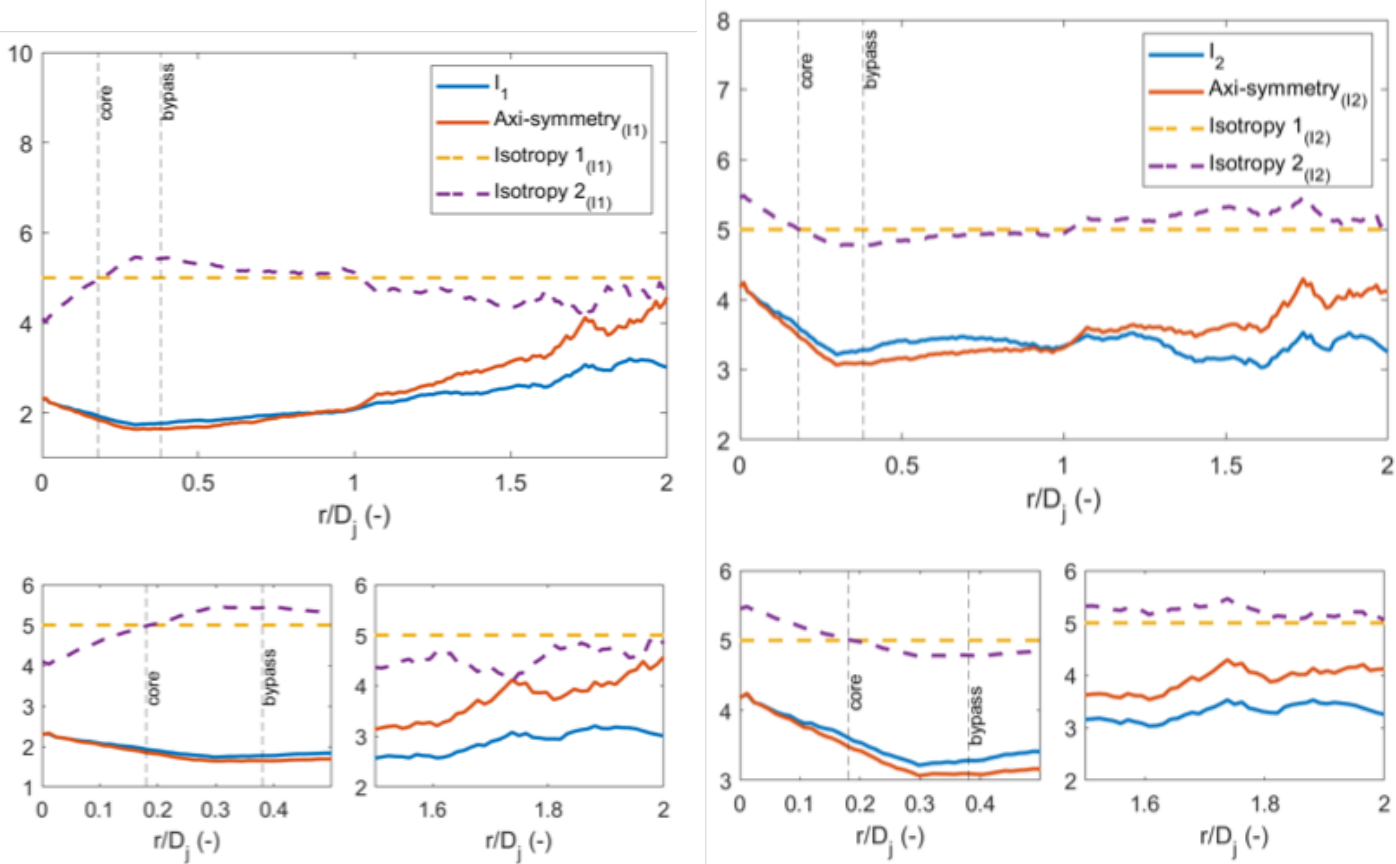

(c) Invariant 1: OP1.7

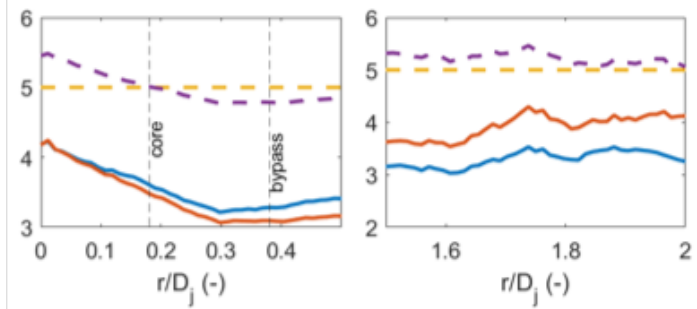

(d) Invariant 2: OP1.7

Fig. 7 Radial variation of invariants 1 and 2 (Fig. 2) for OP1.3 and 1.7 far downstream at $x / D_{j}=10$. 

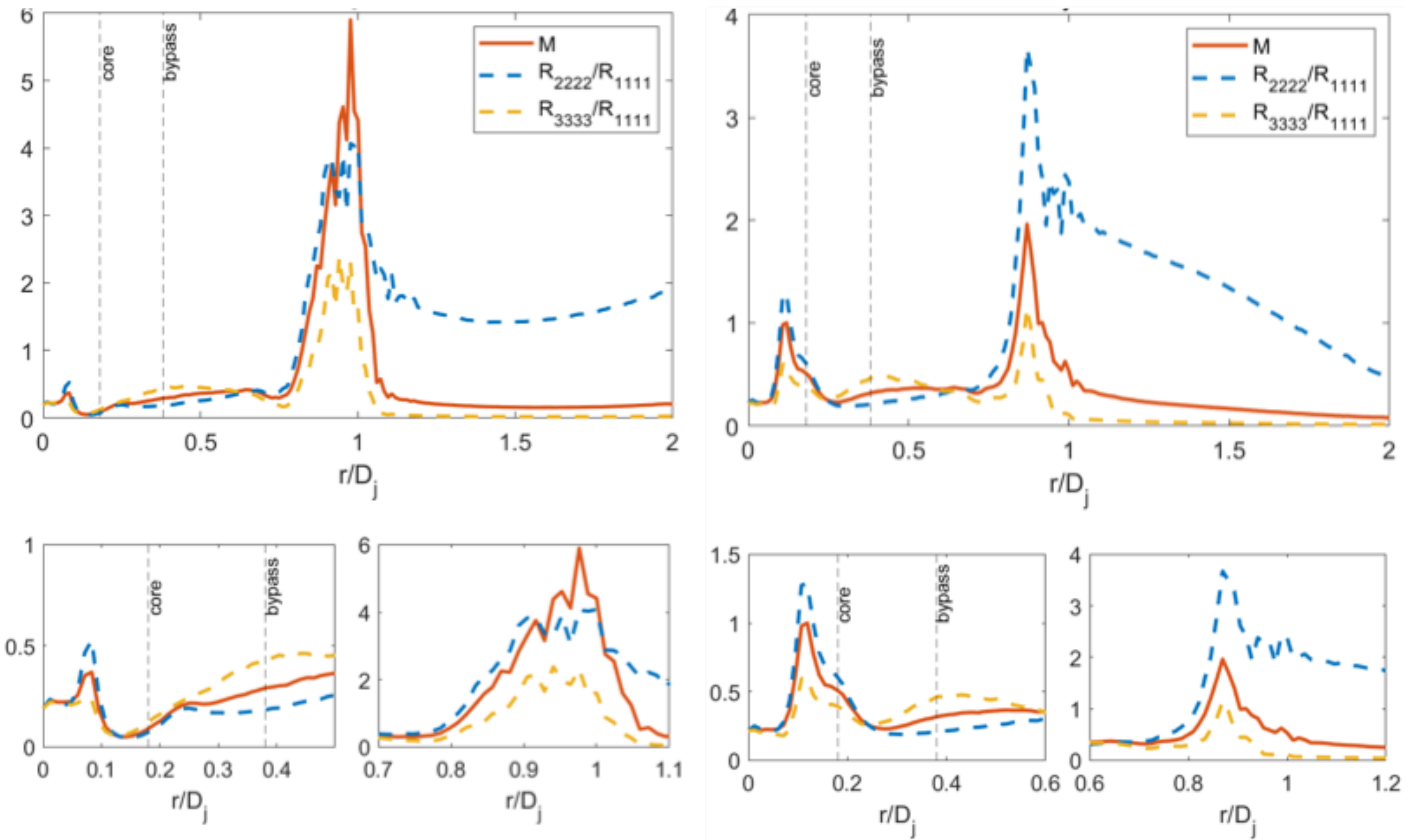

(a) $x / D_{j}=2$ : OP1.3

(b) $x / D_{j}=2$ : OP1.7
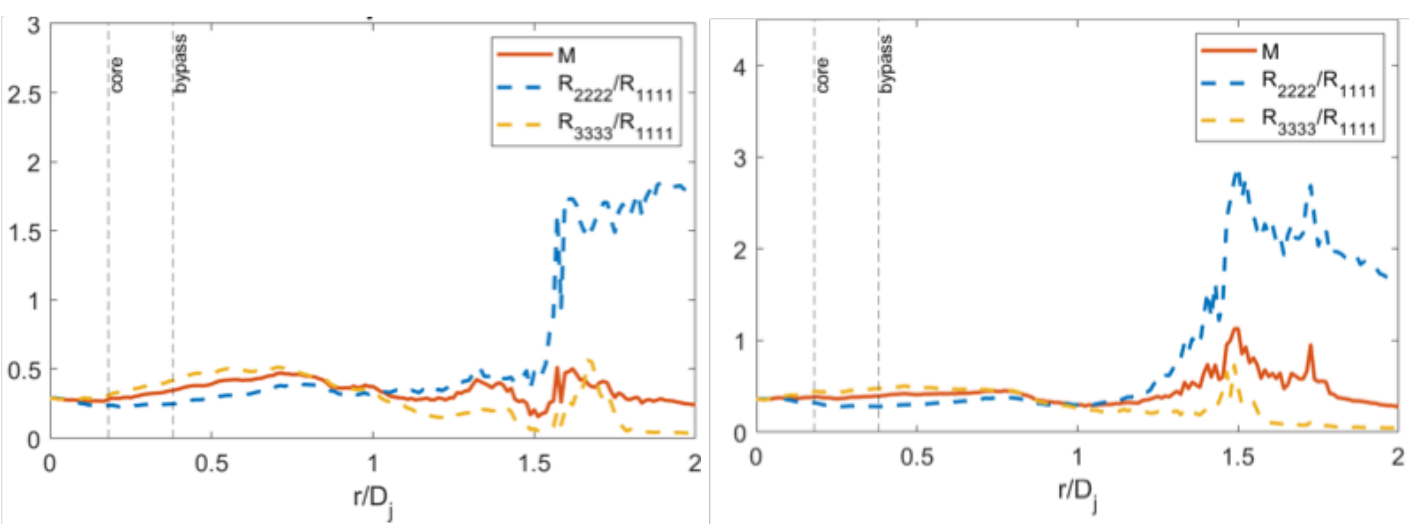

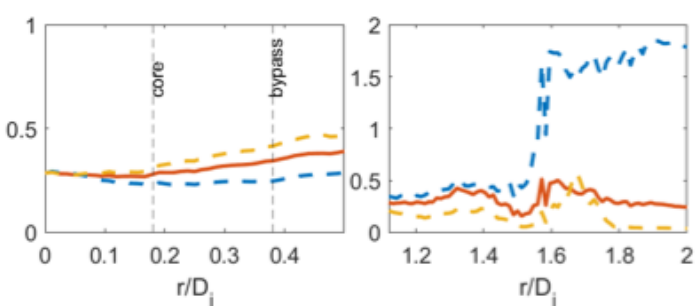

(c) $x / D_{j}=6, \mathrm{OP} 1.3$

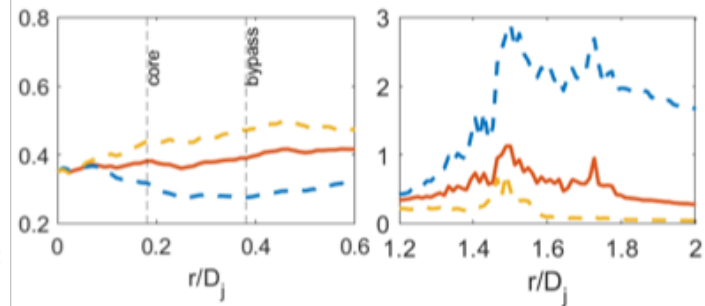

(d) $x / D_{j}=6$, OP1.7

Fig. 8 Radial variation of Millionshchikov identity showing core and bypass regions. 


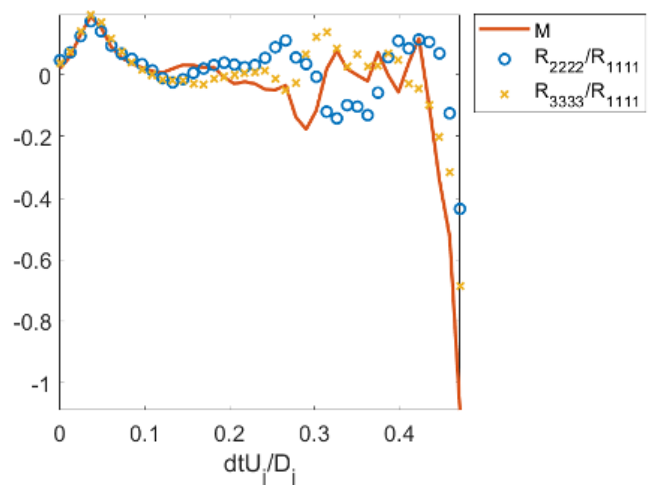

(a) $(x, d x) / D_{j}=(6,0.1)$

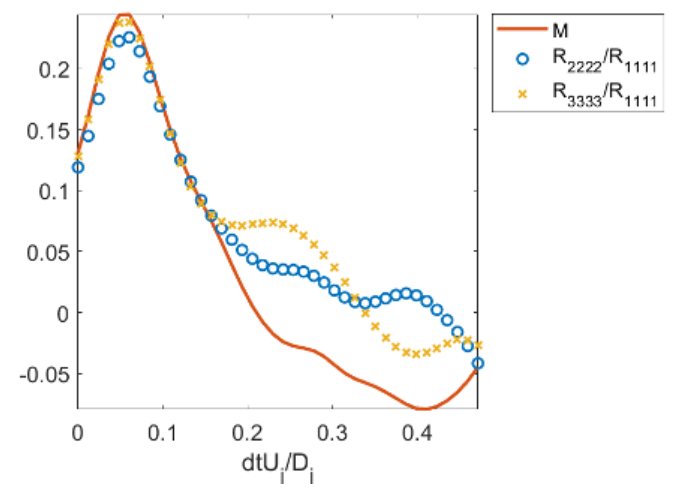

(c) $(x, d x) / D_{j}=(10,0.1)$

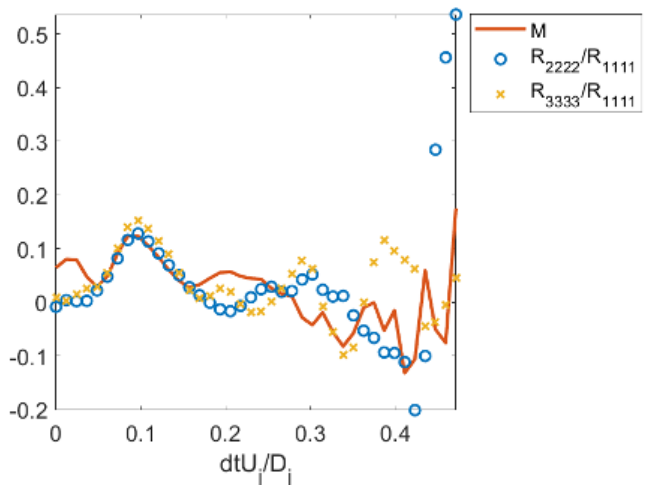

(b) $(x, d x) / D_{j}=(6,0.3)$

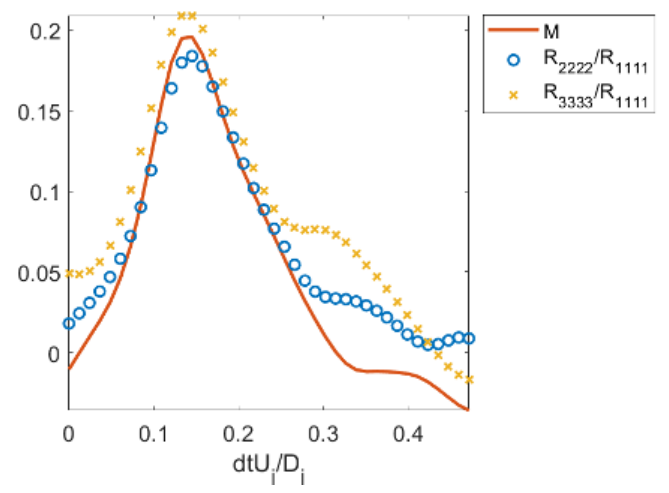

(d) $(x, d x) / D_{j}=(10,0.3)$

Fig. 9 Variation of Millionshchikov identity at fixed spatial separation $d x / D_{j}$ with time delay for OP1.3 in the core region. 


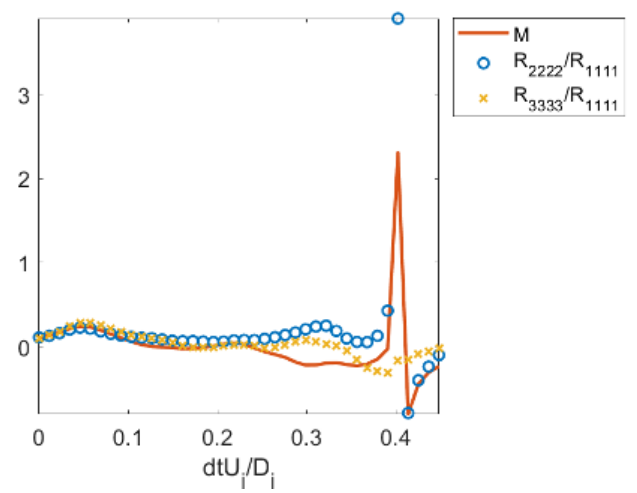

(a) $(x, d x) / D_{j}=(6,0.1)$

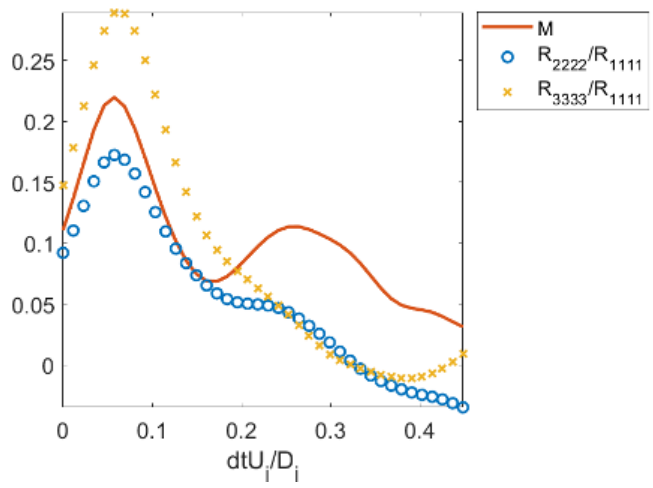

(c) $(x, d x) / D_{j}=(10,0.1)$

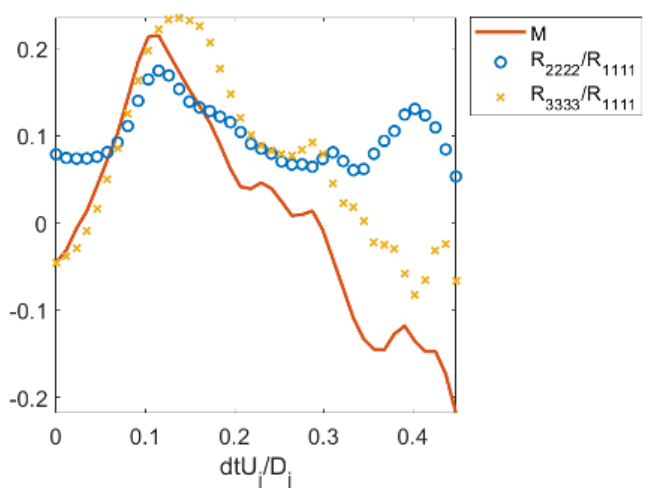

(b) $(x, d x) / D_{j}=(6,0.3)$

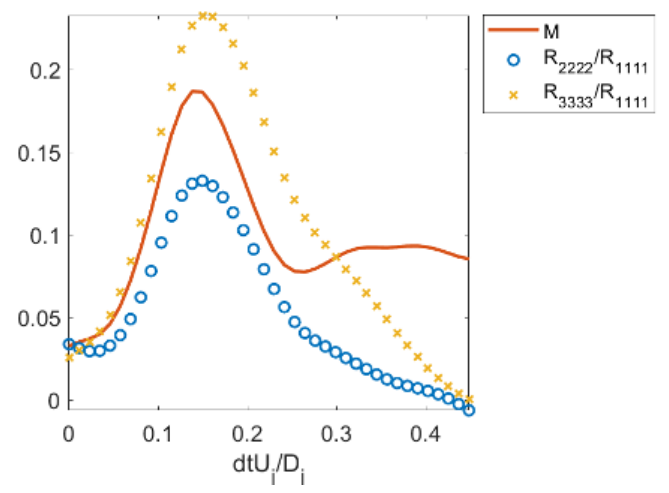

(d) $(x, d x) / D_{j}=(10,0.3)$

Fig. 10 Variation of Millionshchikov identity at fixed spatial separation $d x / D_{j}$ with time delay for OP1.3 in the bypass region. 


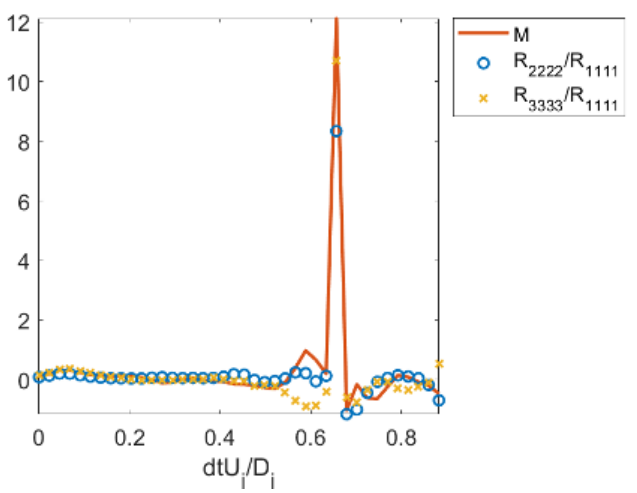

(a) $(x, d x) / D_{j}=(6,0.1)$

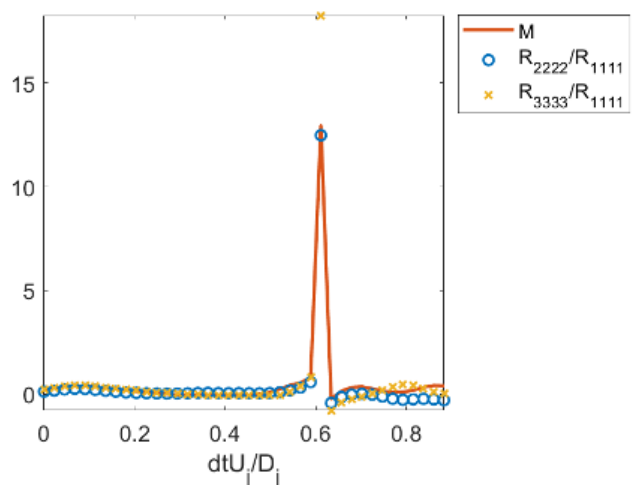

(c) $(x, d x) / D_{j}=(10,0.1)$

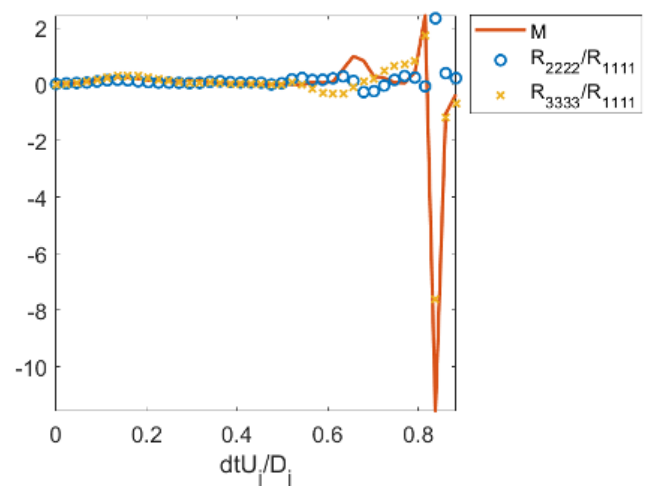

(b) $(x, d x) / D_{j}=(6,0.3)$

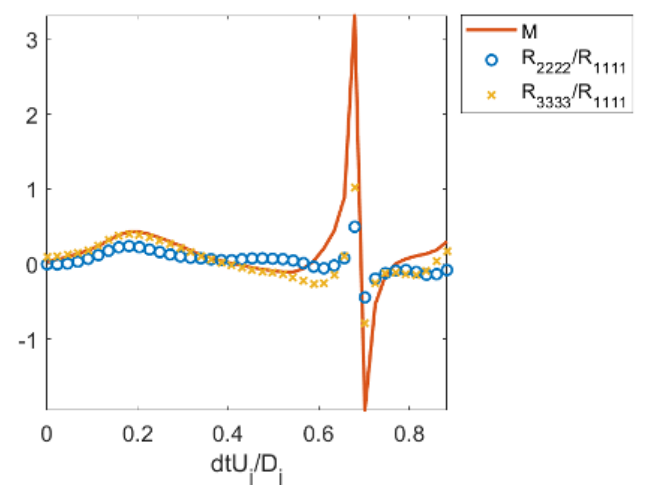

(d) $(x, d x) / D_{j}=(6,0.3)$

Fig. 11 Variation of Millionshchikov identity at fixed spatial separation $d x / D_{j}$ with time delay for OP1.7 in the bypass region. 